\title{
COVID-19 and elderly: beyond the respiratory drama
}

\author{
Angela Sciacqua $^{1,4}$ (D) Roberta Pujia $^{2} \cdot$ Franco Arturi $^{2} \cdot$ Marta L. Hribal $^{2} \cdot$ Tiziana Montalcini $^{3}$
}

Received: 30 April 2020 / Accepted: 26 June 2020 / Published online: 3 July 2020

(c) Società Italiana di Medicina Interna (SIMI) 2020

Dear editor,

Within a very short period, Italy was hit by an urgent health crisis followed by a relentless number of deaths. State health officials and policy makers have been making extraordinary efforts overworking at unprecedented rates on the frontline, to prevent its spread and successfully treat affected individuals. What is currently understood about this global pandemic is that the chances of a COVID-19 patient developing symptoms that are severe enough to require hospitalization and respiratory support increase critically with age. In fact, a higher proportion of deaths have occurred among older people. COVID-19 has killed an estimated 13\% of patients aged 80 and older, compared to $0.3-1.25 \%$ for younger age groups [1]. Infections represent a main cause of death in the elderly. However, chronological age alone does not explain this increase. Researchers agree that in the event of an acute infectious process in the elderly, physicians should look for predisposing factors that could indicate a multifactorial immunodeficiency responsible for the severity of infections and consequent death.

Immunosenescence is an unavoidable process typical of life being. Alterations in innate immunity have a crucial role in this process starting with the reduction of barriers

The article is part of the Topical Collection on COVID-19.

Angela Sciacqua

sciacqua@unicz.it

1 Department of Medical and Surgical Sciences, Geriatric Unit, University Magna Græcia of Catanzaro, Catanzaro, Italy

2 Department of Medical and Surgical Sciences, Internal Medicine Unit, University Magna Græcia of Catanzaro, Catanzaro, Italy

3 Department of Experimental and Clinical Medicine, Clinical Nutrition Unit, University Magna Græcia of Catanzaro, Catanzaro, Italy

4 Department of Medical and Surgical Sciences, Campus Universitario di Germaneto, V.le Europa, 88100 Catanzaro, Italy in the epithelial layer of the skin and gastrointestinal and respiratory mucosa, with a consequent changes in local immunoglobulin ratio. However, the high incidence of infectious events in the elderly can be secondary to alterations in the phagocyte system. Immunosenescence, protein-energy malnutrition (PEM), and the coexistence of multiple diseases and polypharmacy reduce resilience, which means that individuals are less able to cope in the face of adversity or significant sources of stress. Reduced resilience later in life has been associated with several adverse outcomes including immune dysregulation, which could account for a greater susceptibility to COVID-19 infection and a rapid deterioration in clinical conditions (Fig. 1).

Moreover, the social environment may also affect the patient's exposure to infections, with a greater risk for residents in long-term care facilities than for elderly citizens living at home [2].

Although severe respiratory disease is the main clinical manifestation of COVID-19 infection, other important factors may explain the high mortality rate. COVID-19 interacts with the cardiovascular system (CV) on multiple levels, thus negatively influencing the clinical outcome, especially in patients with underlying CV diseases (CVDs). The prevalence of CVDs increases with age, and thus, elderly patients are particularly exposed to acute complications [3] (Fig. 1).

A common condition of old age is a suboptimal diet, which is characterized by inadequate food and calorie intake and an unmet increased protein demand. Malnutrition is prevalent among approximately one-third of older people, especially residents in institutionalized facilities. According to the Global Leadership Initiative on Malnutrition (GLIM) criteria, the top core phenotypic and etiologic criteria to diagnose malnutrition are unintentional weight loss, low body mass index, reduced muscle mass, reduced food intake or assimilation, and disease burden/inflammation (at least one phenotypic criterion and one etiologic criterion are needed to diagnose malnutrition). 
Fig. 1 Mechanisms by which aging and chronic disease lead to infections susceptibility ( $C V D$ cardiovascular disease, $C O P D$ chronic obstructive pulmonary disease, $C K D$ chronic kidney disease)

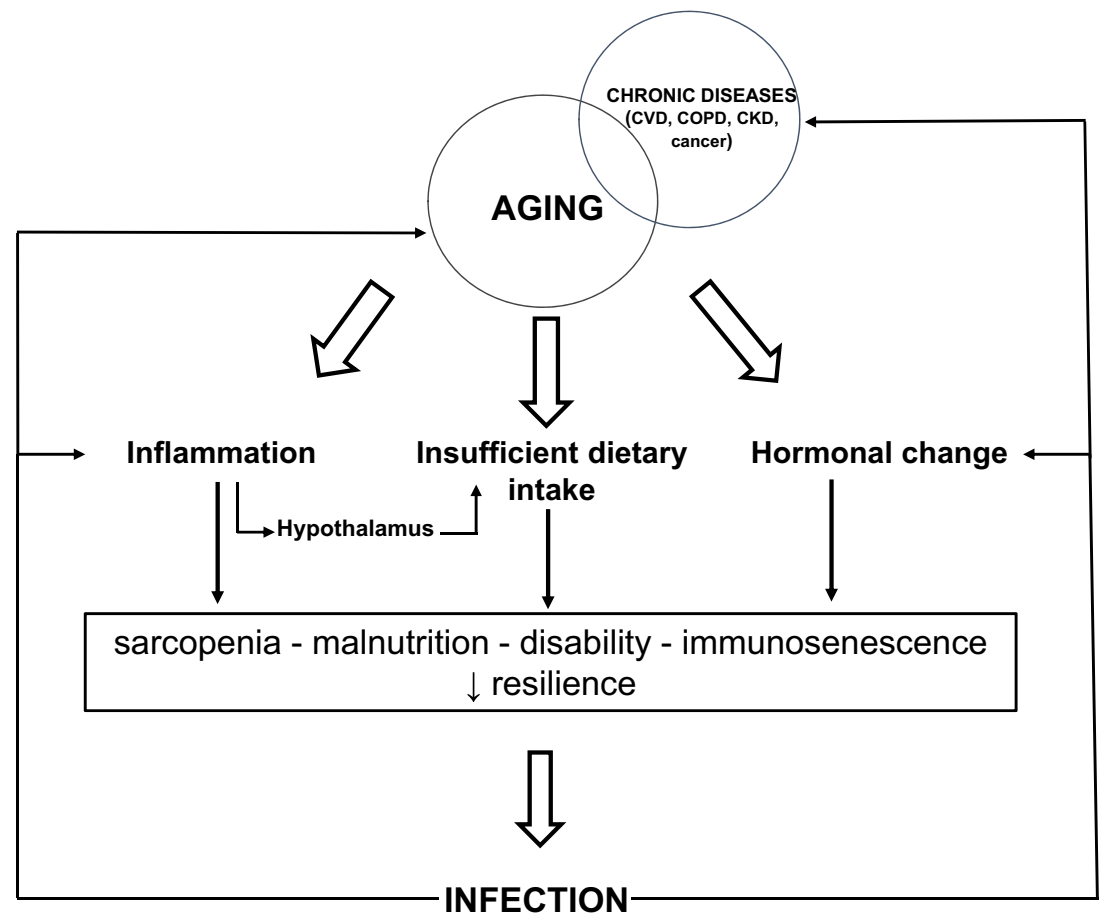

Interestingly, it has been reported that many elderly patients experience a loss of muscle mass and then weight loss, the latter often being stable due to the fat mass increase. When loss of muscle mass is associated with a reduced muscle strength, then an individual is affected by sarcopenia. This clinical condition is the most prevalent syndrome in older medical inpatients.

In its 2018 definition, the European Working Group on Sarcopenia in Older People (EWGSOP) used low muscle strength (a measure of muscle function) as the primary parameter of sarcopenia, which is confirmed by the presence of a low muscle quantity or quality.

Sarcopenia and malnutrition clearly overlap; however, sarcopenia is prevalent in up to $15 \%$ of apparently healthy older adults, up to $80 \%$ of acutely hospitalized older patients, and up to $70 \%$ of older post-acute rehabilitation inpatients. Sarcopenia is associated with CVD, respiratory disease, and cognitive impairment as well as a greater $\mathrm{CV}$ mortality and all-cause mortality [4]. Furthermore, sarcopenic obese individuals, who are those with a reduced muscle mass in the context of excess adiposity, are at higher risk of all-cause mortality [4]. In this respect, several evidences suggest that obesity is associated with a high risk of developing severe symptoms and complications of COVID-19, irrespectively of other clinical conditions.

In today's complex clinical scenario, we believe that it is important to promptly detect the clinical signs indicating malnutrition, sarcopenia, or sarcopenic obesity in elderly patients.
Although a body mass index lower than $25 \mathrm{~kg} / \mathrm{m}^{2}$ is considered a risk factor for mortality in the elderly, this biomarker lacks sensitivity and may not fully reflect the nutritional status. Another biomarker of poor nutritional status is unintentional weight loss. However, assessing the body weight and body weight change is often not feasible in patients with acute clinical conditions. In clinical practice, several plasma proteins are used as indirect markers of PEM. Of these, albumin and prealbumin levels have been advocated as nutritional markers, particularly during refeeding and in the elderly. However, these are unreliable markers of the nutritional status and are good for detecting inflammatory states rather than malnutrition. Another potential marker of nutritional status is the transferrin level, which decreases in cases of severe malnutrition; however, it is unreliable in the assessment of mild malnutrition and in acutely ill elderly patients. Although popular, scientific evidence is inconsistent in terms of proving the validity of serum markers, which are routinely measured, as determinants of patients' nutritional status.

During hospitalization for an acute illness, the instrumental assessment of the loss of muscle mass is clearly not feasible. However, handgrip strength (HGS) is the most widely and reliable method used for the assessment of presarcopenia and it is a significant predictor of morbidity, disability, and mortality in hospitalized elderly patients [5]. A reduction in strength below a certain threshold is predictive of adverse outcomes, such as mortality. Different reference values exist for HGS for different ethnicities. Therefore, a simple HGS test with the appropriate 
cut-off points may be a new paradigm for the clinical risk assessment of complications in the elderly, including those affected by COVID- 19 .

We believe that "active surveillance" in the elderly in long-term care facilities is crucial; however, we believe that it should also be extended to all elderly patients and should include a careful assessment of the nutritional status. All these findings must, therefore, guide toward a new paradigm for the prediction of risk in the elderly:

In the elderly living at home, the evaluation of the nutritional status (clinical examination and at least the HGS measurement) can help to indicate if the elderly person is at risk of getting COVID-19. Although during the acute phase, the priority is to treat the infection, the assessment of the nutritional status during the hospitalization (including body composition) helps to predict the risk of developing complications and mortality and to provide the most appropriate nutritional treatment to restore a good nutritional status. Subsequently, in post-hospitalization, regular nutritional assessments can be used to monitor treatment success.

We believe that the identification of the nutritional predictors of infection susceptibility and adverse outcomes in the elderly could be useful for clinicians on the frontline, such as interns, geriatricians, nutritionists, as well as infectious disease specialists. This is especially true if research findings are translated into executable standards of care to better tackle future epidemics. Only when we have collected sufficient data, including nutritional parameters, to define the scientific basis of the link between aging and mortality from COVID-19, will we be able to develop standard protocols for treatment. Until then, all proposals will be of an empirical nature.

To date, we know that three elements are essential in the dietary treatment of sarcopenia: proteins, vitamin D, and antioxidants. Nutritional advice tailored on an individual basis should be given at an early stage to help prevent a rapid decline in clinical conditions. Elderly individuals require 1.4-2 g/Kg/day of protein. Branched chain amino acids and leucine, in particular, have an important role in muscle protein synthesis, thanks to their high digestibility. Micronutrient intake, especially of vitamin D, could be adjusted in line with the clinical and biochemical profile. Finally, oral nutritional supplements can be beneficial when a normal balanced diet cannot be tolerated.

At the conclusion of the on-going nutritional trials, we will know if all these nutrients are also effective for COVID19 patients.

In conclusion, the global response to COVD-19 must be directed towards those groups who face the most devastating consequences, particularly the elderly. So what can we do?
First, medical schools should focus more on nutrition in their curricula and in the education and training of healthcare professionals in the field of food science and nutrition. Other system-level approaches should also remove obstacles to obtaining food, oral nutritional supplementation, and other essential supplies for elderly in the different clinical settings.

All elderly people must actively monitored, which included systematic nutritional assessments by experienced health care personnel together with the prevention of PEM by dietary/nutritional interventions, also considering vitamin and micronutrient deficiencies. In the meantime, all the necessary data must be collected to clarify, as fully as possible, the pathophysiological basis of the susceptibility of the elderly.

\section{Compliance with ethical standards}

Conflict of interest The authors declare that they have no conflict of interest.

Statement of human and animal rights This article does not contain any studies with human participants or animals performed by any of the authors.

Informed consent For this type of study, formal consent is not required.

\section{References}

1. Garg S, Kim L, Whitaker M, O'Halloran A et al (2020) Hospitalization rates and characteristics of patients hospitalized with laboratory-confirmed coronavirus disease 2019-COVIDNET, 14 States, March 1-30, 2020. MMWR Morb Mortal Wkly Rep 69:458-464

2. Yoshikawa TT, Reyes BJ, Ouslander JG (2019) Sepsis in older adults in long-term care facilities: challenges in diagnosis and management. J Am Geriatr Soc 67:2234-2239

3. Clerkin KJ, Fried JA, Raikhelkar J et al (2020) Coronavirus disease 2019 (COVID-19) and cardiovascular disease. Circulation. https://doi.org/10.1161/CIRCULATIONAHA.120.046941

4. Atkins JL, Whincup PH, Morris RW et al (2014) Sarcopenic obesity and risk of cardiovascular disease and mortality: a population-based cohort study of older men. J Am Geriatr Soc 62:253-260

5. Martìn-Ponce E, Hernàndez-Betancor I, Gonzàlez-Reimers E et al (2014) Prognostic value of physical function tests: hand grip strength and six-minute walking test in elderly hospitalized patients. Sci Rep 22:7530

Publisher's Note Springer Nature remains neutral with regard to jurisdictional claims in published maps and institutional affiliations. 